\title{
Morphological Evolvement of Carbon Nanotubes Synthesized by Using Conducting Polymer Nanofibers
}

\author{
Yang Liu $\mathbb{D}^{1}{ }^{1}$ John H. Xin, ${ }^{2}$ Xinyu Zhang $\mathbb{D}^{3},{ }^{3}$ and Chao Zhang $\mathbb{D}^{1}$ \\ ${ }^{1}$ Department of Biomedical Engineering, Sun Yat-sen University, Guangzhou, China 510006 \\ ${ }^{2}$ Institute of Textiles and Clothing, The Hong Kong Polytechnic University, Hung Hom, Hong Kong, China
}

${ }^{3}$ Department of Chemical Engineering, Auburn University, Auburn, Alabama, USA 36849

Correspondence should be addressed to Yang Liu; liuyang56@mail.sysu.edu.cn

Received 20 September 2019; Revised 12 December 2019; Accepted 18 February 2020; Published 2 March 2020

Academic Editor: Christopher Batich

Copyright ( 2020 Yang Liu et al. This is an open access article distributed under the Creative Commons Attribution License, which permits unrestricted use, distribution, and reproduction in any medium, provided the original work is properly cited.

Carbon nanotubes were synthesized by using a nanostructured conducting polymer-the polypyrrole nanofiber via microwave radiation. The radiation time was set to be 30,60, and 90 seconds, respectively. The morphological evolvements of the assynthesized carbon nanotubes with increased radiation time (e.g., shape, diameter, wall structure, and catalyst size) were carefully investigated, and the possible growth mode was discussed in detail. It was found that the growth mode of the carbon nanotubes synthesized from the conducting polymer substrate under microwave radiation was complex and cannot be simply interpreted by either a "tip" or "base" growth model. A new growth mode of the "liquifying cascade growth" was observed for the as-synthesized carbon nanotubes, as their growth was directed by a series of liquified iron nanoparticles with sequentially decreasing sizes, similar to the cascade of liquid droplets. And it could provide useful insights for the morphological and structural designs of the carbon nanotubes prepared by related microwave-based methods.

\section{Introduction}

Carbon nanotubes (CNTs) represent a special onedimensional allotrope of graphite with distinct thermal, electrical, mechanical, and chemical properties [1]. Currently, CNTs are majorly synthesized by thermal techniques such as chemical vapor deposition (CVD), arc discharge (AC), and laser ablation (LA) [2]. However, the CVD techniques majorly use the gasified hydrocarbons as the precursor while $\mathrm{AC}$ and LA use the decomposed carbonaceous species generated from high-energy sources as precursors. Recently, extensive research efforts have been made to develop the synthesis technique of CNTs based on conducting polymers [3-10]. Due to their excellent microwave absorption properties, conducting polymers, e.g., polypyrrole (Ppy), can be used as the substrate to grow CNTs in a domestic microwave oven. Upon microwave radiation, the conducting polymer quickly absorbs the electromagnetic waves and elevates the reaction temperature to several hundreds of degrees Celsius. This triggers the decomposition of ferrocene to generate iron $(\mathrm{Fe})$ nanoparticles as the catalyst and gaseous hydrocarbons as the carbon source to initiate the CNT growth. Due to the highly efficient heating of the conducting polymer, the whole CNT growth process can be completed within tens of seconds $[3,6,9,10]$. Compared to CVD, AC, and LA techniques, the conducting polymer-based microwave technique may attain the characteristics of simple instrumentation, easy-processing, and very fast growth. It can also be used to grow CNT brushes on the surface of different materials, e.g., carbon fiber fabrics, MXene, Kevlar fibers, and poly(lactic-co-glycolic acid) particles to obtain CNT-based hierarchical composites with enhanced electrochemical, mechanical, and bioimaging properties $[3,9,11,12]$. Even though the growth of CNTs by using the conducting polymer-based microwave technique is simple and fast, currently, there are very few reports on the morphological study of the as-obtained CNTs. And the mechanisms involved in the microwave growth process have not been comprehensively studied and are still unclear.

The morphological control of CNTs is very important because the size and shape of CNTs can significantly affect their properties and functions [13]. For example, the bamboo-shaped CNTs were reported to have high energy- 
storage performance such as high-discharge capacities, high rate capabilities, and low potential gaps, as they were used as the electrode catalysts of the $\mathrm{Li}-\mathrm{CO}_{2}$ batteries [14]. On the other hand, it was also reported that the bambooshaped CNTs showed exponential decrease of scission length and low tensile strengths during ultrasonication [15]. Coiled CNT forests also showed strongly nonlinear dependence on displacement which was found to be fundamentally different from the response of a straight CNT forest, under high-strain rate deformation of a drop-ball test [16]. The morphology of the as-synthesized CNTs is intrinsically clung to the growth modes. Currently, either a "tip growth" or "base growth" model has been proposed to the CNTs synthesized by using the conducting polymer-based microwave techniques, deducing from the location of the catalyst nanoparticles (NPs) [3, 6]. However, these assumptions are rather empirical and insufficient to describe the internal growth modes of CNTs synthesized by the conducting polymer microwave technique according to our experimental observations.

Herein, we carefully investigate the growth mode of the CNTs synthesized by using conducting polymer, i.e., polypyrrole nanofibers (Ppy NFs), under the microwave radiation of a domestic microwave oven. The radiation time was gradually increased to study the morphological evolvement of the CNTs in the process. Compared to the nonnanostructured conducting polymers that are widely used to grow CNTs, the Ppy NFs would have stronger microwave absorbing properties due to their one-dimensional (1D) nanostructure and high surface area [17]. The correlation between the CNT morphology and the morphology of the catalyst NPs is also discussed in detail. And a new possible growth mode of the CNTs synthesized by conducting polymer-based microwave techniques is proposed.

\section{Experimental}

2.1. Synthesis of CNTs by Using Ppy NFs and Ferrocene. Ppy NFs were synthesized by a modified "seeding approach" [17]. Briefly speaking, $1 \mathrm{~mL}$ pyrrole (Alfa Aesar, 98\%) was added into $60 \mathrm{~mL} 1 \mathrm{M} \mathrm{HCl}$ and magnetically stirred for 20 minutes (min). Subsequently, $1 \mathrm{~mL}$ vanadium pentoxide $\left(\mathrm{V}_{2} \mathrm{O}_{5}\right)$ nanofiber sol-gel was injected into the dispersion quickly by using a $1 \mathrm{~mL}$ syringe. The transparent dispersion turned light green immediately, and Ppy NFs were starting to precipitate out of the dispersion as $\mathrm{V}_{2} \mathrm{O}_{5}$ was added. After 30 seconds (s), $1.09 \mathrm{~g}$ initiator ammonium persulfate (Alfa Aesar, 98\%) was added to trigger the polymerization process. The polymerization was proceeded for another 1 hour under magnetic stirring. After polymerization, the black precipitation was suction filtered and washed with copious amounts of $1 \mathrm{M} \mathrm{HCl}$ and acetone to remove the impurities. The asobtained Ppy NFs were dried in an $80^{\circ} \mathrm{C}$ oven overnight before further usage.

$70 \mathrm{mg}$ of Ppy NFs and $70 \mathrm{mg}$ ferrocene (Alfa Aesar, 99\%) were mechanically mixed using an agate mortar and then transferred to a $20 \mathrm{~mL}$ quartz-glass vial. The black powder of Ppy NF was thoroughly mixed with the yellowish ferrocene, and the final mixture appeared as homogeneous black powders. The glass vial was then placed into a domestic microwave oven (Panasonic Inverter 1000) and heated at $1000 \mathrm{~W}$ power for different durations, i.e., $30 \mathrm{~s}$, $60 \mathrm{~s}$, and $90 \mathrm{~s}$. As the microwave radiation was applied, the black mixture was quickly heated up and turned red, which was similar to the color of hot iron, due to the intensive absorption of microwave by the Ppy NFs. At the same time, the temperature of the mixture was quickly risen to several hundreds of degrees Celsius and triggered the decomposition of ferrocene, generating high density of gaseous carbonaceous species floating inside the glass vial and covering its inner walls. As the microwave process continued, intensive microplasmas were generated near the surface of the mixture, which interacted with the gaseous species to give shining sparks inside the glass vial. Simultaneously, high pressure was generated by the hot gas inside the vial, and the shape of the quartz glass was also slightly deformed. The microwave heating process was automatically stopped when the preset time was depleted. The glass vial was then cooled in ambient condition, and the product was subsequently collected and purified by washing with copious amounts of toluene and water to remove the residues of precursors.

2.2. Characterizations of the as-Synthesized CNTs. The morphologies of the as-synthesized CNTs were characterized by a scanning electron microscope (SEM, JEOL JXA-8600). The tube-wall and graphitic structures of the CNTs were characterized by a transmission electron microscope (TEM, JEOL JEM-2100F) equipped with an energy dispersive Xray detector (EDX, Oxford Instruments INCA X-Max). The TEM sample was prepared by adding $3 \mathrm{mg}$ of the assynthesized CNTs in $5 \mathrm{~mL}$ de-ionized water, which was subsequently bath-sonicated for $20 \mathrm{~min}$ to form a homogeneous dispersion. One or two drops from the dispersion was then casted on a TEM copper grid by using a glass pipette. The elemental composition and crystalline structure of the catalysts were analyzed by EDX and selected area electron diffraction (SAED).

The size distributions of the as-synthesized CNTs were measured by analyzing the corresponding SEM and TEM images using the ImageJ software. For each set of the samples, at least 50 different CNTs were counted and analyzed to calculate the values of average diameter and standard deviation, respectively.

\section{Results and Discussion}

The representative SEM images of the CNTs synthesized by using Ppy nanofibers and ferrocene under different microwave radiation time are shown in Figure 1. Apparently, thin and curved CNTs are obtained from the time of $30 \mathrm{~s}$, as shown in Figures 1(a) and 1(b). The CNTs are radially grown outward from many granulated micron-sized particulates, resembling the structure of a sea urchin (Figure 1(a)). As the microwave time increases to $60 \mathrm{~s}$, significant changes in the morphology can be observed for the as-obtained CNTs, compared to the $30 \mathrm{~s}$ situation. In this case, thicker and straighter CNTs are grown from the granulated substrates, and the radial growth is disturbed (Figures $1(\mathrm{c})$ and $1(\mathrm{~d})$ ). The as-grown CNTs tend to randomly entangle with each 


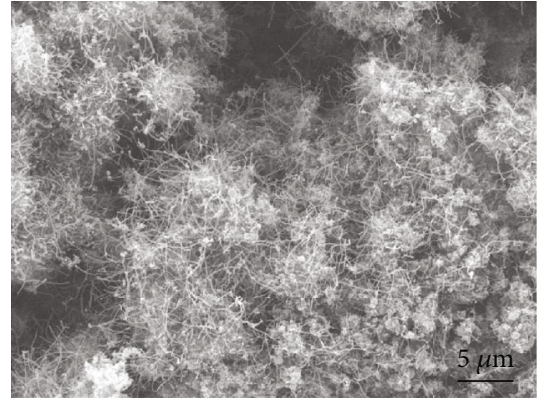

(a)

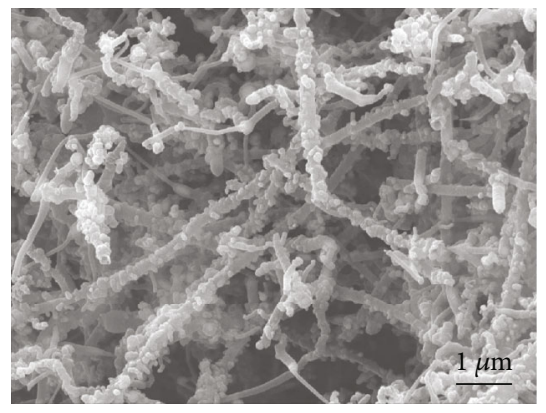

(d)

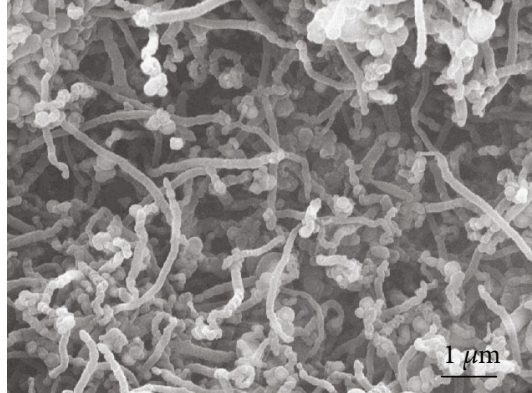

(b)

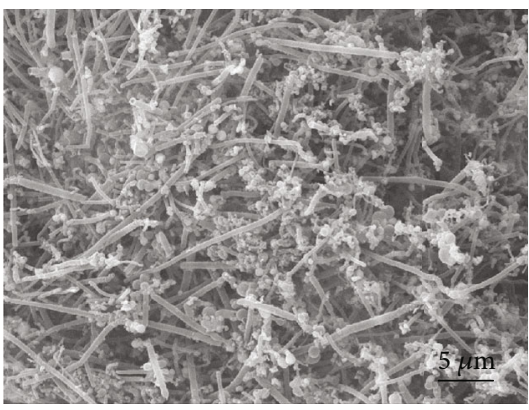

(e)

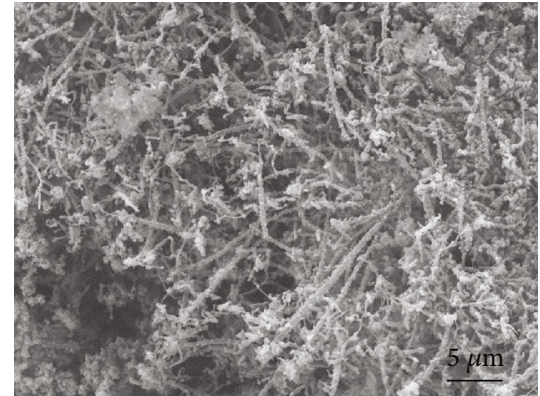

(c)

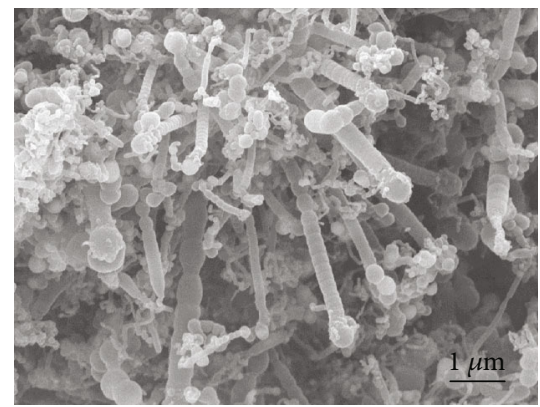

(f)

Figure 1: SEM images of CNTs synthesized by Ppy NFs and ferrocene under microwave radiation for $30 \mathrm{~s}(\mathrm{a}, \mathrm{b}), 60 \mathrm{~s}(\mathrm{c}, \mathrm{d})$, and $90 \mathrm{~s}(\mathrm{e}, \mathrm{f})$.

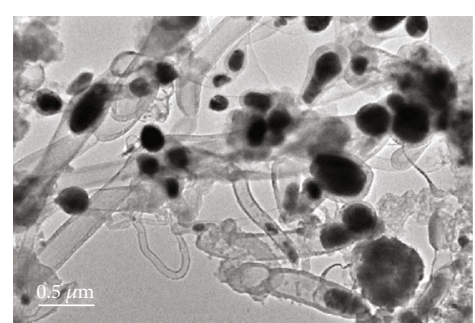

(a)

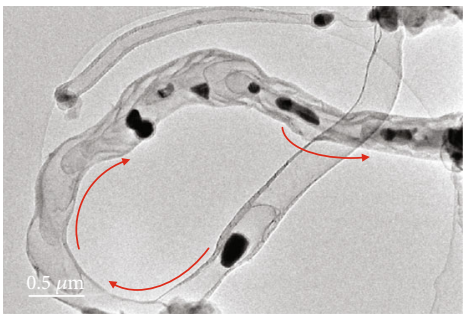

(d)

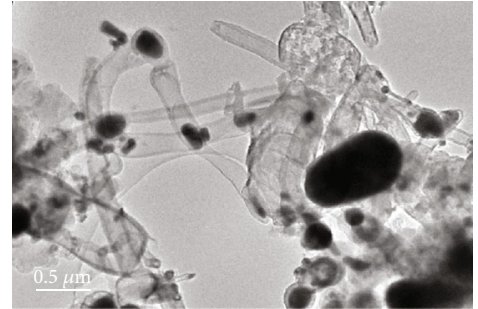

(b)

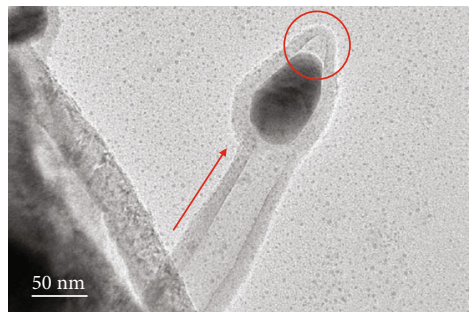

(e)

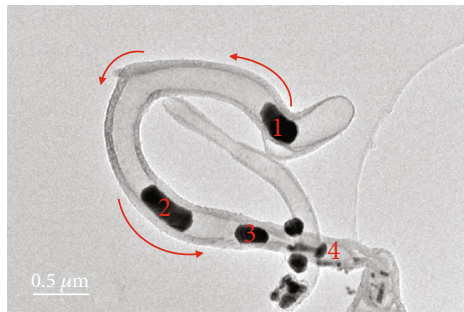

(c)

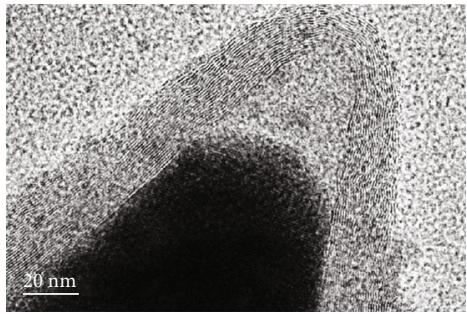

(f)

Figure 2: $(a, b)$ TEM images of the CNTs synthesized by 30 s microwave time and protruded catalyst Fe NPs are observed to direct the CNT growth. (c, d) TEM images showing the cascade growth process of the CNTs; the catalyst Fe NPs are protruded towards the growth direction and lifted to direct the growth from the parent NP, forming a discontinuous cascade of NPs. The growth direction is marked with red arrows. (e) TEM image showing a CNT as the growth process is terminated. A catalyst Fe NP encapsulated in carbon shells can be observed at the tip of the CNT. The carbon shells are composed of orderly stacking graphene layers as shown in (f).

other, forming porous networks. Upon further increasing the microwave time to $90 \mathrm{~s}$, thick and straight CNTs are found to grow from the granulated substrates at various angles, while a portion of thin and curved CNTs are also observed to exist alongside with them (Figure 1(e)). The other portion of the thick and straight CNTs obtained from $90 \mathrm{~s}$ show a characteristic bamboo-shaped morphology, as the side walls of the CNTs are apparently segmented into periodic nanoscale compartments (Figure 1(f)). The average diameters of the CNTs obtained from $30 \mathrm{~s}$ are calculated to be $145.8 \mathrm{~nm}$, with a standard deviation of $18.7 \mathrm{~nm}$. And the calculated average diameters of the CNTs obtained from $60 \mathrm{~s}$ and $90 \mathrm{~s}$ are $268.8 \mathrm{~nm}$ and $356.8 \mathrm{~nm}$, with standard deviations of $56.8 \mathrm{~nm}$ and $141.2 \mathrm{~nm}$, respectively.

The TEM images of CNTs obtained from $30 \mathrm{~s}$ are shown in Figure 2. From Figure 2(a), it can be observed that the tube 


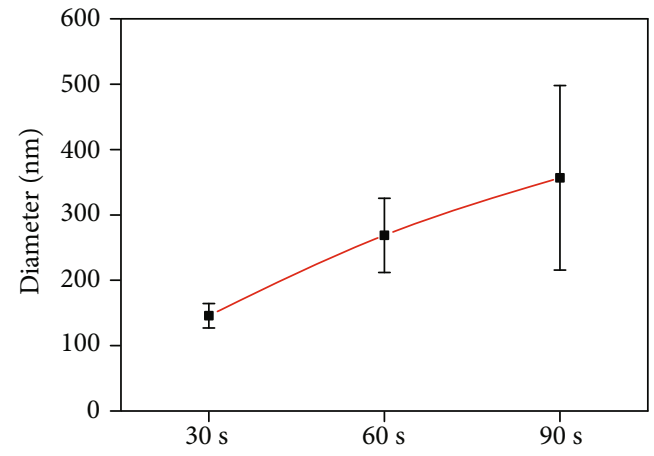

(a)

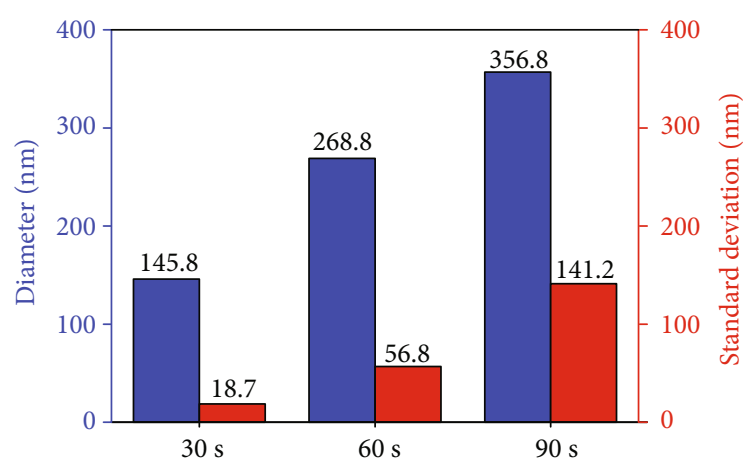

(b)

Figure 3: (a) Plotted graph showing the correlation between the average CNT diameters and microwave time; the standard deviations are plotted as the error bars. (b) Columns showing the direct comparison of the average diameters and corresponding standard deviations of the CNTs synthesized from $30 \mathrm{~s}, 60 \mathrm{~s}$, and $90 \mathrm{~s}$.

walls of the as-obtained CNTs are generally smooth, composing of continuous stacking graphene layers with structural homogeneity. Catalyst NPs can be observed inside the hollow cores of the CNTs, and they are identified to be the polycrystalline Fe NPs according to the EDX (Figure S1) and the SAED analysis (Figure S2). The shapes of the Fe NPs are ellipsoid, spherical, and ellipsoid with one protruded end. The average diameters of the Fe NPs are measured to be $136.6 \mathrm{~nm}$ with a standard deviation of $51.9 \mathrm{~nm}$. And the average outer diameters of the corresponding CNTs are measured to be $139.9 \mathrm{~nm}$, indicating a strong correlation between the NP diameter and the CNT diameter. The CNTs are found to preferentially nucleate and grow on smaller $\mathrm{Fe}$ NPs at the early stage, while larger NPs (diameters $>200 \mathrm{~nm}$ ) show a slower CNT growth kinetics in terms of the length and thickness of the forming tube walls. A few large Fe NPs with diameters between 260 and $440 \mathrm{~nm}$ also can be observed. These large Fe NPs are partially covered by multiple layers of graphene sheets with one end protrudes and points towards the propagating CNT growth direction (Figures 2(a) and 2(b)). It is speculated that the Fe NPs are partially liquified to form the protruding ends during the growth process. The liquified portion of the NPs then serve as the active catalytic center to direct the CNT growth. As the carbon atoms continuous diffuse and precipitate out from the liquified NPs, the protruding portion of the $\mathrm{Fe}$ NPs may detach from the original NP and lift off. The CNTs then continue to grow from the lifting liquified Fe NPs, producing smooth tube walls whose inner diameters are identical to the minor axis of the liquified NPs (Figure 2(c)). At this time, the lifting liquified NPs may act as the active tips to direct the CNT growth. However, the lifted liquified NPs may receive fewer thermal energies as they move away from the heating substrates, and the lifting process as a whole is stopped due to the partial cooling and solidifying of the NPs. In this case, a smaller liquified NP may again protrude from the parent NP and lifted to direct the CNT growth, repeating the previous growth process. The growing of CNTs would be stopped if the lifted NPs become too small or solidified, as shown in Figures 2(c) and 2(d). Either tip or base growth mode has been suggested for the CNTs synthesized from ferrocene under microwave radiation, using conducting polymers or graphite as the heating substrate. This is due to the fact that the Fe NPs can be observed to distribute along the major tube axis of the as-obtained CNTs, e.g., at the tips, at the bottoms, and in the middle. This phenomenon actually reveals the liquifying cascade growth mode of the CNTs under microwave which cannot be simply interpreted as tip or base growth, because the Fe NPs in the liquid state may solidify and split into multiple smaller NPs to direct the tube growth. As the liquified Fe NPs solidify, thick carbon shells with orderly stacking graphene layers are formed along the surface profile of the NPs, as shown in Figures 2(e) and 2(f). The tip of the Fe NP is capped with closed carbon shells, and no further growth can be initiated, indicating the termination of the growth process (Figure 2(f)). Stacking faults and misalignments can be observed in the tube wall structures of the CNTs obtained from 30 s (Figure S3), indicating the reduced degree of graphitization in short microwave time.

The changes in the diameters of the CNTs synthesized by using different microwave time were firstly analyzed through the SEM images. The quasilinear increase in the average diameters with prolonged microwave time of the as-obtained CNTs indicates their growth process is kinetically controlled (Figure 3(a)). The rate determine step is speculated to be the decomposition of ferrocene. The standard deviations to the average diameters significantly increase with prolonged microwave time, indicating the CNTs synthesized from shorter time would have a more uniform diameter distribution and longer microwave time may generate a wide-range diameter distribution for the CNTs that confers significant differences in diameters between the individual CNTs (Figure 3(b)). However, the increase of CNT diameters, significantly widened diameter distributions, and the emerging of CNTs with extremely large diameters as the microwave time is prolonged all in total reflect the change of the growth kinetics as it proceeds. Indeed, the total morphological evolvement of the CNTs is directly controlled by the growth kinetics and can be simply modulated by tuning the microwave time. On the other hand, the growth kinetics, including carbon diffusion, metal-carbon interaction, and carbon wall structure formation, are strongly related to the dynamic crystalline structures of the metal 


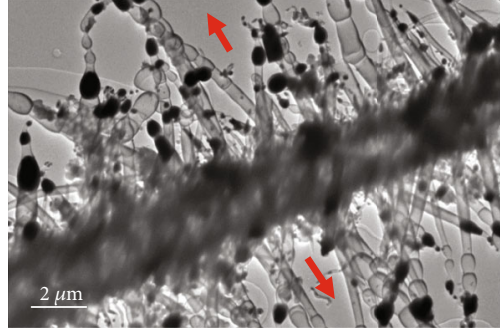

(a)

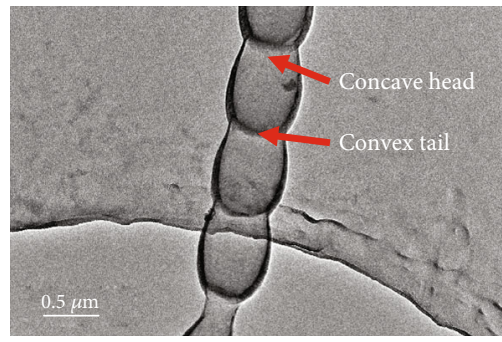

(d)

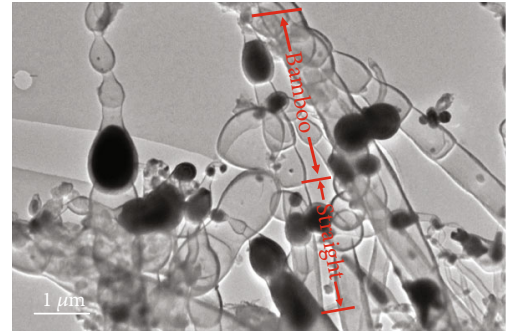

(b)

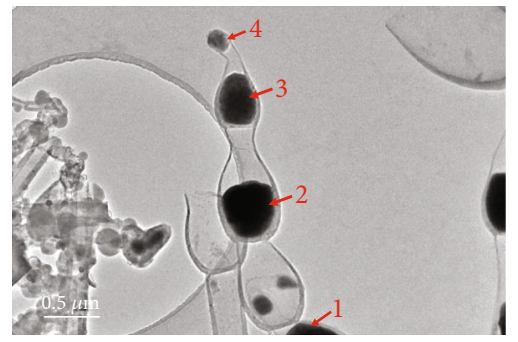

(e)

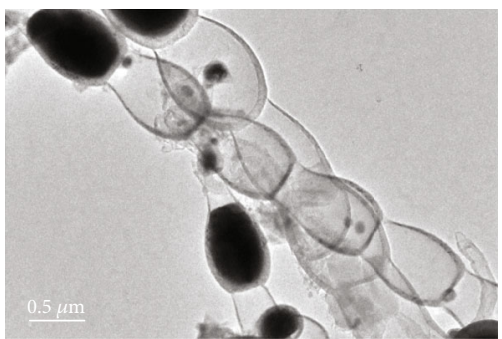

(c)

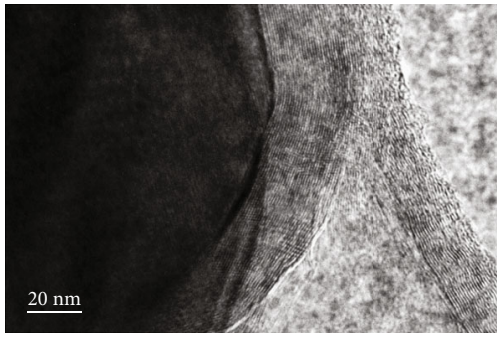

(f)

FIgURE 4: (a, b) TEM images of the bamboo-shaped CNTs synthesized by $60 \mathrm{~s}$ microwave time. The bamboo-shaped CNTs are found to grow in opposite directions from the sheet-like substrate in (a), and the growth direction is marked with red arrows. The bamboo-shaped CNTs are found to compose of one straight part with smooth and continuous tube wall and the other part with bamboo-like segmented tube walls, as shown in (b). (c, d) TEM images showing the magnified view of the bamboo-shaped CNTs. The catalyst Fe NPs can be observed at the tips of the CNTs, as shown in (c). And the growth direction of the bamboo-shaped CNTs is identified to be from the convex tails to the concave heads, as shown in (d). (e) Liquifying cascade growth mode similar to the $30 \mathrm{~s}$ situation is also observed for the $60 \mathrm{~s}$ CNTs. The cascading catalyst NPs with reducing sizes are numbered with arithmetic orders. (f) HRTEM image showing the well-stacking graphitized tube walls of the bamboo-shaped CNTs.

NPs that are acting as the catalysts. By investigating the size and shape changes of the metal NPs during the CNTs' growth and the influences of these changes on the CNTs' morphologies, we can gain useful insights on the detail growth processes, growth modes, and microstructural formation of the as-grown CNTs.

As the microwave time increases to $60 \mathrm{~s}$, significant morphological changes are observed for the as-obtained CNTs. Both CNTs with smooth and continuous tube walls (Figure S4) and CNTs with bamboo-like wall structures (Figure 4(a)) are found to coexist in the products. The average diameters of the CNTs measured from the TEM images are $246 \mathrm{~nm}$, and this value is considered to be consistent with the SEM analysis. The bamboo-like CNTs are observed to grow from the same sheet-like substrate (Figure 4(a)). The CNTs are grown perpendicularly to the substrate in two opposite directions at different angles. The catalyst Fe NPs are found to intensively exist inside the tube cores, lifted towards the tips, forming cascade NPs of decreasing sizes within single bamboo-like CNTs, which are similar to the liquid droplet cascades. By carefully examining the bamboo-like CNTs, it is found that they are generally composed of a smooth and straight tube portion connecting to a bamboo-shaped portion (Figure 4(b)). This abrupt change of the tube structure can be possibly attributed to the phase transition of the catalyst Fe NPs. It has been reported that Ni catalyst NPs underwent a reversible phase change from the liquified state to the solidified state in the temperature range from 800 to $700^{\circ} \mathrm{C}$, resulting in a structure change of the CNT tube walls from straight to cup-stack [18]. It has also been reported that the as-grown CNTs would obtain a tube wall structure reproducing the shape of the active catalyst NPs [19]. Therefore, in the first place, the liquified Fe NPs may extrude from the heating substrate and direct the CNT growth. At this stage, CNTs with smooth and continuous tube walls can be formed. Due to the unique heating mechanism of microwave, the heats are generated by the microwave absorbing substrates, i.e., Ppy nanofiber. As the catalyst Fe NPs move away from the substrate, they receive fewer thermal energies and become reluctant to maintain the liquid-like form. The slow cooling of the Fe NPs results in the gradual shrinkage of their shapes from elongated liquified states to solidified spherical states, and it is measured to happen when the NPs are 0.89$3.17 \mu \mathrm{m}$ distant from the substrate, with an average distance of $1.81 \pm 0.54 \mu \mathrm{m}$, according to the TEM images. During the shrinkage, the NPs may still acquire the partial liquified state and mobile to direct the CNT growth until completely cooling. Therefore, a general trend that can be observed for the growth of bamboo-like CNTs is the length and size of the individual bamboo-like compartments decrease as the tube length increases, coincided with the phase change of the catalyst Fe NPs. The bamboo-like CNTs are formed by the stacking of several cup-shaped compartments with convex tails and concave heads, and the catalyst Fe NPs can be found around the tips of the CNTs (Figure 4(c)). By identifying the locations of the convex tails and the concave heads, the growth direction of the bamboo-like CNTs can be manifested (Figure 4(d)). The CNTs obtained from $60 \mathrm{~s}$ share the same growth mode with the $30 \mathrm{~s}$, and the sequence 


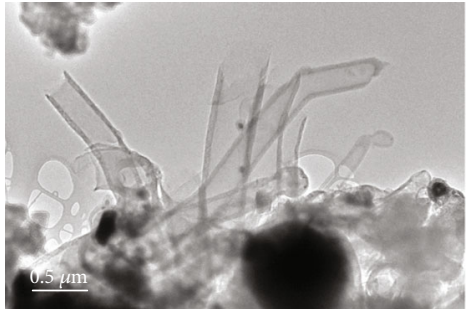

(a)

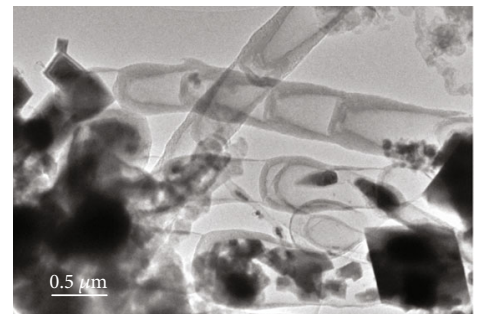

(d)

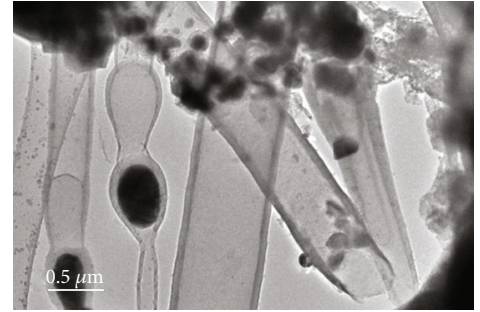

(b)

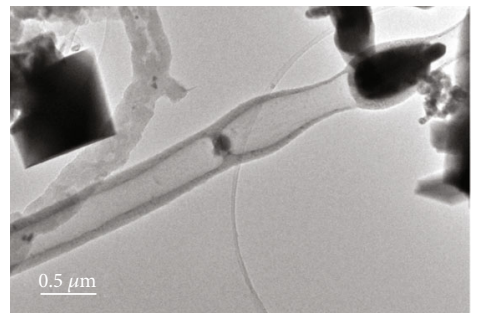

(e)

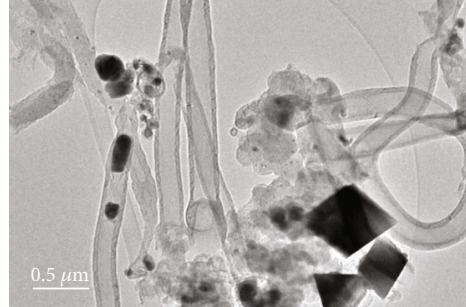

(c)

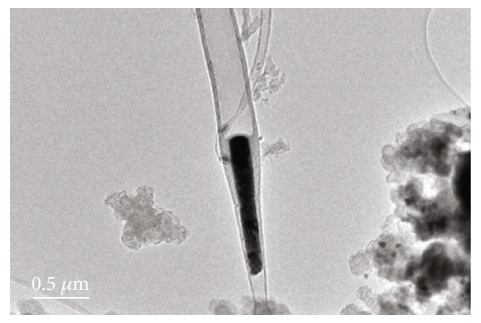

(f)

FIGURE 5: Different morphologies can be observed for the CNTs synthesized by 90 s microwave time, such as straight tubes with continuous tube walls (a), bamboo-shaped tubes with segmented tube walls (b), curved tubes with continuous tube walls (c), and cup-stack tubes (d). A few large rhombohedral and rectangular NPs can be observed in (c), (d), and (e), indicating substantial Ostwald ripening of the catalysts. Protruding (e) and liquid-shaped (f) catalyst Fe NPs can be observed at the tips of the as-obtained CNTs, indicating the same growth mode with the $30 \mathrm{~s}$ and $60 \mathrm{~s}$ processes.

of the cascading NPs can be clearly observed around their tips (Figure 4(e)). The $60 \mathrm{~s}$ CNTs exhibit well-graphitized wall structure with a lattice spacing of $0.34 \mathrm{~nm}$ without stacking faults both on and parallel to the catalyst surface, indicating the growth time is sufficient to achieve a higher degree of graphitization (Figure 4(f) and Figure S5).

As the microwave time increases to $90 \mathrm{~s}$, the as-obtained CNTs show diverse morphologies including straight, curved, bamboo-shaped, and cup-stack (Figures 5(a)-5(d)). The average diameter of the CNTs obtained from the TEM analysis is calculated to be $324.9 \mathrm{~nm}$, close to the SEM analysis result. The as-obtained CNTs also showed orderly stacking graphitic shells, with larger shell thickness than the $60 \mathrm{~s}$ tubes (Figure S6). However, the substantial inhomogeneity observed in the morphologies for the $90 \mathrm{~s}$ CNTs can be possibly attributed to the inhomogeneous nucleation and growth processes of the catalyst Fe NPs which produces NPs with a wide range of size distribution to catalyze the CNT growth. It has been reported that the size of the catalyst NPs would continue growing during the CNT growth process by both surface diffusion and the Ostwald ripening process [20]. The surface diffusion of the catalyst atoms onto the same catalyst NPs may result in the size increment of the NPs and eventually deactivate the catalyst NPs to grow CNTs [21]. On the other hand, the Ostwald ripening process would result in the continuous growth of the large catalyst NPs by consuming the smaller ones. The combination of these two effects may give rise to significant differences in size between the large and small catalyst NPs. Interestingly, remarkable differences in size can be observed for the catalyst Fe NPs during the $90 \mathrm{~s}$ growth. The large Fe NPs with sizes from 190 to $513 \mathrm{~nm}$ and small NPs with sizes from 74 to $160 \mathrm{~nm}$ are found to coexist in the core regions of the CNTs (Figures 5(b)-5(e)). A few large NPs with rhombohedral and rectangular shapes can also be observed around the CNT bundles (Figures 5(c)-5(e)). There are no graphitic shells covered on the surface of these large NPs, indicating that they are inert to catalyze the CNT growth. Interestingly, a rhombohedral NP is observed at the tip end of a bamboo-shaped CNT, and the smaller NPs in the compartment of the CNT are merging towards the rhombohedral NP, which can be taken as an indication for the Ostwald ripening process (Figure 5(d)). Besides the remarkable Ostwald ripening phenomenon on the catalyst NPs, the CNTs obtained from 90 s show a similar growth mode to the $30 \mathrm{~s}$ and $60 \mathrm{~s}$ situations, as the liquifying catalyst Fe NPs elongated and elevated from the parent NPs to direct the CNT growth and subsequently form a liquifying cascade of smaller NPs to continue the growth process (Figures 5(e) and 5(f)). Eventually, the growth process would be stopped as the small NPs were deactivated by the surface-covered graphitic shells (Figure S7).

\section{Conclusions}

In this article, we detailedly investigated the morphological evolvement of the CNTs synthesized by using Ppy nanofibers and ferrocene under microwave radiation. The reaction kinetics of the growth process were elaborated by analyzing the morphologies of CNTs and catalyst Fe NPs obtained from increased microwave time, i.e., $30 \mathrm{~s}, 60 \mathrm{~s}$, and $90 \mathrm{~s}$. The diameters of CNTs and their degrees of graphitization were found to simultaneously increased with prolonged microwave time from $30 \mathrm{~s}$ to $90 \mathrm{~s}$. A strong correlation was also found between the diameters of the CNTs and sizes of the catalyst NPs. By carefully analyzing the TEM images of the catalyst NPs inside the tubes, a new liquifying cascade growth mode was proposed for the as-obtained CNTs. Due to the 
unique heating mechanism of microwave, the liquified catalyst NPs would receive fewer thermal energies as they were elevating from the substrates (heat source) and became gradually solidified. During this stage, a morphological transformation from smooth and straight tubes to bamboo-shaped tubes can be observed. However, the growth process of CNTs was continued until the cascade liquified NPs became fully covered by the graphitic shells or too small to catalyze the CNT formation. The Ostwald ripening of the catalyst NPs was found to become significant as the microwave time was increased to $90 \mathrm{~s}$. The remarkable difference in size between the large and small NPs and a wide-range size distribution of the catalyst NPs lead to a highly divergent diameter distribution of the as-obtained CNTs, from approximately $180 \mathrm{~nm}$ to $740 \mathrm{~nm}$, after the $90 \mathrm{~s}$ microwave process. A few rhombohedral NPs with sizes from around $200 \mathrm{~nm}$ to $480 \mathrm{~nm}$ were discovered near the tips of the CNTs. These NPs were inert for catalyzing the CNT growth, and it was speculated that they were composed of trigonal hematite crystals according to their shapes.

To further improve the morphological homogeneity of the CNTs synthesized by conducting polymers and ferrocene under microwave, a more homogeneous heating mechanism may need to be implemented to eliminate the thermal gradient near the surface of the substrate and help the NPs maintain their liquified states, e.g., additional microwave absorbing materials like carbon fibers can be introduced on the substrate's surface. On the other hand, the Ostwald ripening process may also need to be suppressed during the growth. To achieve this goal, the microwave synthesis process can be conducted in more reducing environment with the addition of reducing agents such as hydrogen gas.

\section{Data Availability}

The data used to support the findings of this study are included within the article and the supporting information files.

\section{Conflicts of Interest}

The authors declare no conflict of interests.

\section{Acknowledgments}

The financial supports from Sun Yat-sen University, National Natural Science Foundation of China (81801851), Guangdong Science and Technology Department (2018A030310075), and China Postdoctoral Science Foundation (2018M633230) were gratefully acknowledged.

\section{Supplementary Materials}

The supporting information file contains the experimental results, including the transmission electron microscopy (TEM) images, high-resolution TEM images (HRTEM), energy-dispersive X-ray (EDX) spectrum, and selected-area electron diffraction (SAED) pattern, to support the discussions in the manuscript. Figure S1: EDX spectrum of the catalyst nanoparticle observed inside the core of the as-obtained
CNTs; the elemental composition of the nanoparticle was identified to be Fe. Figure S2: SAED pattern of a catalyst Fe NP encapsulated at the tip of a CNT. The interpretation of pattern indicated the NP was polycrystalline. Figure S3: $(a, c)$ TEM and (b, d) HRTEM images of the CNTs synthesized by $30 \mathrm{~s}$ microwave time. The representative stacking faults are marked with blue arrows. Figure S4: (a-d) TEM images of the CNTs with smooth and continuous tube walls synthesized by $60 \mathrm{~s}$ microwave time. Figure S5: (a, c, e) TEM and (b, d, f) HRTEM images of the CNTs synthesized by $60 \mathrm{~s}$ microwave time. A lattice spacing of $0.34 \mathrm{~nm}$ can be observed for the graphitic tube walls. Figure S6: $(a, c, e)$ TEM and the corresponding ( $b, d, f)$ HRTEM images of the CNTs synthesized by $120 \mathrm{~s}$ microwave time. Figure S7: HRTEM image of a Fe catalyst nanoparticle encapsulated by graphitic shells. (Supplementary Materials)

\section{References}

[1] Y. R. Poudel and W. Li, "Synthesis, properties, and applications of carbon nanotubes filled with foreign materials: a review," Materials Today Physics, vol. 7, pp. 7-34, 2018.

[2] G. D. Nessim, "Properties, synthesis, and growth mechanisms of carbon nanotubes with special focus on thermal chemical vapor deposition," Nanoscale, vol. 2, no. 8, pp. 1306-1323, 2010.

[3] Z. Liu, J. Wang, V. Kushvaha et al., "Pop-tube approach for ultrafast carbon nanotube growth," Chemical Communications, vol. 47, no. 35, pp. 9912-9914, 2011.

[4] S. H. Bae, K. Karthikeyan, Y. S. Lee, and I. K. Oh, "Microwave self-assembly of 3D graphene-carbon nanotube-nickel nanostructure for high capacity anode material in lithium ion battery," Carbon, vol. 64, pp. 527-536, 2013.

[5] V. Sridhar, I. Lee, H. H. Chun, and H. Park, "Microwave synthesis of nitrogen-doped carbon nanotubes anchored on graphene substrates," Carbon, vol. 87, pp. 186-192, 2015.

[6] R. Bajpai and H. D. Wagner, "Fast growth of carbon nanotubes using a microwave oven," Carbon, vol. 82, pp. 327-336, 2015.

[7] S. K. Park, K. Choi, S. H. Lee, I. K. Oh, S. Park, and H. S. Park, "CNT branching of three-dimensional steam-activated graphene hybrid frameworks for excellent rate and cyclic capabilities to store lithium ions," Carbon, vol. 116, pp. 500-509, 2017.

[8] R. Kumar, R. K. Singh, D. P. Singh et al., "Synthesis of selfassembled and hierarchical palladium-CNTs-reduced graphene oxide composites for enhanced field emission properties," Materials \& Design, vol. 122, pp. 110-117, 2017.

[9] W. Zheng, P. Zhang, J. Chen, W. B. Tian, Y. M. Zhang, and Z. M. Sun, "In situsynthesis of CNTs@Ti3C2hybrid structures by microwave irradiation for high-performance anodes in lithium ion batteries," Journal of Materials Chemistry A, vol. 6, no. 8, pp. 3543-3551, 2018.

[10] Y. Liu, X. Zhang, S. Poyraz, C. Zhang, and J. H. Xin, “One-step synthesis of multifunctional zinc-iron-oxide hybrid carbon nanowires by chemical fusion for supercapacitors and interfacial water marbles," ChemNanoMat, vol. 4, no. 6, pp. 546-556, 2018.

[11] A. Hazarika, B. K. Deka, D. Y. Kim, K. Kong, Y. B. Park, and H. W. Park, "Microwave-synthesized freestanding ironcarbon nanotubes on polyester composites of woven Kevlar fibre and silver nanoparticle-decorated graphene," Scientific Reports, vol. 7, no. 1, 2017. 
[12] H. Xie, S. Poyraz, M. Thu et al., "Microwave-assisted fabrication of carbon nanotubes decorated polymeric nano-medical platforms for simultaneous drug delivery and magnetic resonance imaging," RSC Advances, vol. 4, no. 11, p. 5649, 2014.

[13] M. Zhang and J. Li, "Carbon nanotube in different shapes," Materials Today, vol. 12, no. 6, pp. 12-18, 2009.

[14] X. Li, J. Zhou, J. Zhang et al., "Bamboo-like nitrogen-doped carbon nanotube forests as durable metal-free catalysts for self-powered flexible Li-CO2Batteries," Advanced Materials, vol. 31, no. 39, article 1903852, 2019.

[15] J. W. Jang, C. E. Lee, and C. J. Lee, "Exponential decrease of scission length and low tensile strength of bamboo- shaped multi-walled carbon nanotubes under ultrasonication," Current Applied Physics, vol. 17, no. 4, pp. 507-512, 2017.

[16] C. Daraio, V. F. Nesterenko, S. Jin, W. Wang, and A. M. Rao, "Impact response by a foamlike forest of coiled carbon nanotubes," Journal of Applied Physics, vol. 100, no. 6, article 064309, 2006.

[17] X. Zhang and S. K. Manohar, "Bulk synthesis of polypyrrole nanofibers by a seeding approach," Journal of the American Chemical Society, vol. 126, no. 40, pp. 12714-12715, 2004.

[18] E. F. Kukovitsky, S. G. L'vov, N. A. Sainov, V. A. Shustov, and L. A. Chernozatonskii, "Correlation between metal catalyst particle size and carbon nanotube growth," Chemical Physics Letters, vol. 355, no. 5-6, pp. 497-503, 2002.

[19] N. Q. Zhao, C. N. He, J. Ding et al., "Bamboo-shaped carbon nanotubes produced by catalytic decomposition of methane over nickel nanoparticles supported on aluminum," Journal of Alloys and Compounds, vol. 428, no. 1-2, pp. 79-83, 2007.

[20] S. Sakurai, M. Inaguma, D. N. Futaba, M. Yumura, and K. Hata, "Diameter and density control of single-walled carbon nanotube forests by modulating Ostwald ripening through decoupling the catalyst formation and growth processes," Small, vol. 9, no. 21, pp. 3584-3592, 2013.

[21] M. J. Bronikowski, P. A. Willis, D. T. Colbert, K. A. Smith, and R. E. Smalley, "Gas-phase production of carbon single-walled nanotubes from carbon monoxide via the HiPco process: a parametric study," Journal of Vacuum Science and Technology $A$, vol. 19, no. 4, pp. 1800-1805, 2001. 